MISCELLANEOUS

- 76 • 


\section{Constrictive Pericarditis in Isolation}

\section{Dr. Sunil C. Jha}

This is a case of a young female patient with clinical signs of mitral stenosis. First echocardiography was negative for mitral stenosis and chest roentgenogram showed calicification over the edge of left atrium which led to a second echocardiogram. There was constrictive pericarditis at the left atrio ventricular groove mimicking the signs of mitral stenosis. Constrictive pericarditis involving the pericardium overlying the left atrium is rare, Review of literature revealed only one case reported so far. Case is presented here. 